\title{
Existence of chaos in the Chen system with linear time-delay feedback
}

\author{
Kun Tian, Hai-Peng Ren* \\ Shaanxi Key Laboratory of Complex System Control and Intelligent Information Processing \\ Xi'an University of Technology, Xi'an, 710048, China \\ 1075103282@qq.com, renhaipeng@xaut.edu.cn ${ }^{*}$ \\ Celso Grebogi \\ Xi'an University of Technology, Xi'an, 710048, China, \\ Institute for Complex System and Mathematical Biology, \\ Aberdeen, AB24 3UE, United Kingdom \\ grebogi@abdn.ac.uk
}

Received (to be inserted by publisher)

\begin{abstract}
It is mathematically challenging to analytically show that complex dynamical phenomena observed in simulations and experiments are truly chaotic. The Shil'nikov Lemma provides a useful theoretical tool to prove the existence of chaos in three-dimensional smooth autonomous systems. It requires, however, the proof of the existence of a homoclinic or heteroclinic orbit, which remains a very difficult technical problem if contigent on data. In this paper, for the Chen system with linear time-delay feedback, we demonstrate a homoclinic orbit by using a modified undetermined coefficient method and we propose a spiral involute projection method. In such a way, we identify experimentally the asymmetrical homoclinic orbit in order to apply the Shil'nikov-type Lemma and to show that chaos is indeed generated in the Chen circuit with linear time-delay feedback. We also identify the presence of a single-scroll attractor in the Chen system with linear time-delay feedback in our experiments. We confirm that the Chen single-scroll attractor is hyperchaotic by numerically estimating the finite-time local Lyapunov exponent spectrum. By means of a linear scaling in the coordinates and the time, such a method can also be applied to the generalized Lorenz-like systems. The contribution of this work lies in: first, we treat the trajectories corresponding to the real eigenvalue and the image eigenvalues in different ways, which is compatible with the characteristics of the trajectory geometry; second, we propose a spiral involute projection method to exhibit the trajectory corresponding to the image eigenvalues; third, we verify the homoclinic orbit by experimental data.
\end{abstract}

Keywords: linear time-delay feedback; Shil'nikov-type lemma; homoclinic orbit; single-scroll hyperchaotic attractor.

\section{Introduction}

Large number of chaotic phenomena have been observed in physical experiments and in simulations. However, because the amount of data obtained in experiments are typically limited and simulation data have

*Corresponding author:Hai-Peng Ren,renhaipeng@xaut.edu.cn 
finite precision, such observations may not prove to be sufficient to show the existence of chaos in the dynamics of the system under consideration. Thereby, a theoretical proof of the presence of chaos in an experiment is of great significance though difficult. The famous Lorenz system had been studied as a typical example of chaos systems for more than 40 years, but it was shown to be truly chaotic by using a computer-aid proof only rather recently [Tucker, 1999].

Shil'nikov proved that if there exists a homoclinic or heteroclinic orbit in the dynamics of a system and a certain eigen-condition is satisfied, then the system has a Smale horseshoe [Shil'nikov, 1965]. Therefore, it has chaos in a mathematical sense [Shil'nikov, 1965; Silva, 1993; Huang \& Yang, 2005]. In recent years, research on homoclinic and heteroclinic orbits in dynamical systems has attracted much attention [Ren \& Li, 2010; Lv \& Tang, 2013; Chen, 2013; Li et al., 2013; Costa \& Tehrani, 2014; Balasuriya \& PadbergGehle, 2014; Lima \& Teixeira, 2013; Zhou et al., 2004; Wang et al., 2007; EI-Dessoky et al., 2012; Zheng \& Chen, 2006]. The study about the homoclinic and heteroclinic orbits in second-order differential systems has received major development [Lv \& Tang, 2013; Chen, 2013; Li et al., 2013; Costa \& Tehrani, 2014; Balasuriya \& Padberg-Gehle, 2014; Lima \& Teixeira, 2013]. Reference [Zhou et al., 2004] determined the homoclinic and heteroclinic orbits in the Chen system by using the undetermined coefficient method, which was also applied to the Lorenz-family system [Wang et al., 2007], Lü system, Zhou's system [EI-Dessoky et al., 2012], a class of 3D quadratic autonomous chaotic systems [Zheng \& Chen, 2006] and the Chen system with time-delays [Ren \& Li, 2010]. But that effort did not resolve the problem of asymmetry by time-reversal, i.e., the problem of mixed time-reversibility [Algaba et al., 2010, 2012, 2013a,b, 2014]. A new method suggested in [Bao \& Yang, 2011] transformed time into the logarithmic scale so as to avoid converting an orbit in the infinite time-domain into a boundary value problem. A noteworthy result is that an approximate homoclinic orbit can be obtained by choosing the intermediate parameter [Leonov, 2013a,b; Leonov et al., 2015a]. Xie [Xie, 2014] discussed the issue of existing heteroclinic orbits connecting saddle foci, which, however, does not guarantee the existence of analytical solutions. By modifying the boundary condition, homoclinic or heteroclinic orbits can be determined by predicting the orbital direction [Dong \& Lan, 2014], which is difficult to implement in practical situations. A blow-up technique was also presented to obtain an exact homoclinic connection [Algaba et al., 2015]; however, it is complicated to locate the homoclinic orbits using this technique. Chen et al. improved the perturbation method based on nonlinear time transformation to achieve explicit homoclinic solutions, but it can only deal with the powerlaw nonlinear oscillator [Chen et al., 2017]. By finding the set of pruning domains, Huaraca \& Mendoza identified a finite set of homoclinic orbits, if there exists a pronged singularity without rotation [Huaraca \& Mendoza, 2016]. Particularly, references [Chen et al., 2017] and [Huaraca \& Mendoza, 2016] gave an invariant formal expression, but failed to analytically describe the homoclinic motion for a general system. Lin et al. used Lyapunov-Schmidt reduction and exponential dichotomies to derive general conditions under which the perturbed system have transverse homoclinic solutions, but it did not apply to infinite dimensional system [Lin et al., 2015].

In this paper, we first introduce a new method to experimentally validate the homoclinic orbit of the system, which treats in the time forward and time reversal directions separately. We apply a modified undetermined coefficient method to obtain the part of the orbit corresponding to the real eigenvalue of the saddle point, and then propose a spiral involute projection method to determine the part of orbit corresponding to the complex eigenvalue. We identify a single-scroll chaotic attractor in the non-chaotic Chen system with linear time-delay feedback from an experiment, and our proposed method is successfully applied to identify the homoclinic orbit from experimental data of the single-scroll chaotic attractor. By using the Shil'nikov-type Lemma, we show the existence of chaos in the system. Compared to the existing methods that confuse the time forward and time reversal directions, the proposed method is a general method to show the presence of the Shil'nikov-type homoclinic orbit. To the authors' best knowledge, this method is being used in delay-differential equation for the first time.

The organization of this paper is as follows. In Sec. 2, we present a modified undetermined coefficient method and the spiral involute projection method to identify the homoclinic orbit in the dynamical system. In Sec. 3, we show the experiment results of the single-scroll attractor in the Chen system with linear timedelay feedback. The simulations are used as a guide to implement the proposed techniques for experimental data. Based on that, by using the Shil'nikov-type Lemma, we show that the experimental Chen system 
with linear time-delay feedback is truly chaotic. Conclusions are given in Sec. 4.

\section{The improved analytical homoclinic solutions in the chaotic system}

\subsection{Shil'nikov criterion for homoclinic orbit}

Consider a third-order dynamical system:

$$
\frac{d \mathbf{x}}{d t}=\mathbf{f}(\mathbf{x}), t \in R, \mathbf{x} \in R^{3},
$$

where $\mathbf{f}: R^{3} \rightarrow R^{3}, \mathbf{f} \in C^{r}, r \geq 2$. If there exists $\mathbf{X}_{\mathbf{e}}$ that satisfies $\mathbf{f}\left(\mathbf{X}_{\mathbf{e}}\right)=\mathbf{0}$, then $\mathbf{X}_{\mathbf{e}}$ is an equilibrium of system (1). $\mathbf{X}_{\mathbf{e}}$ is referred to as a hyperbolic saddle foci if the eigenvalue of $\mathbf{X}_{\mathbf{e}}$ satisfy the following Shil'nikov inequalities:

$$
\omega \neq 0, \gamma \sigma<0,|\gamma|>|\sigma|>0
$$

where $\gamma$ is the real eigenvalue, and $\sigma \pm j \omega$ are a pair of complex eigenvalues corresponding to the equilibrium.

In dynamical systems, a heteroclinic orbit is a trajectory in phase space which connects two different equilibrium points. A homoclinic orbit of the system (1) is a trajectory that is asymptotic to the same saddle focus both as $t \rightarrow \infty$ and $t \rightarrow-\infty$, as shown in Fig. 1 .

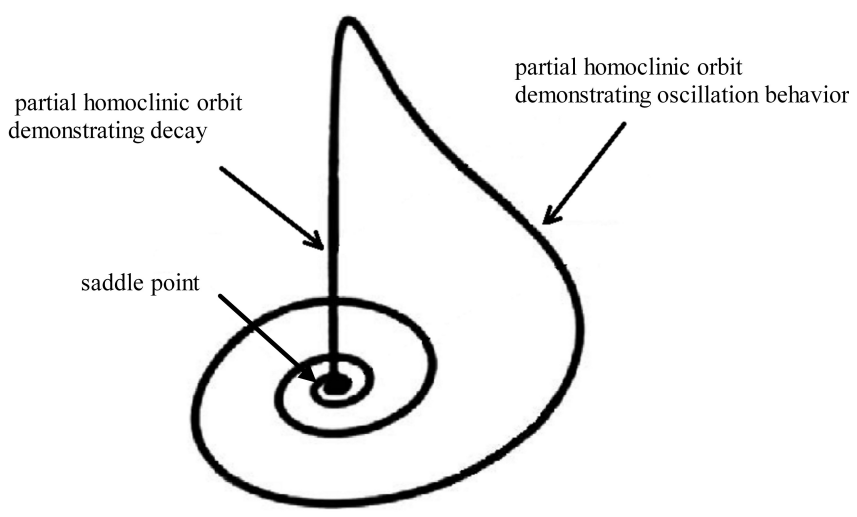

Fig. 1. A homoclinic orbit illustration.

Another necessary concept is that of Poincaré map. A plane denoted by $\Gamma \subset R^{2}$ cuts transversely across the recurrent behavior close to homoclinic or heteroclinic orbit. Define a 2-D map $P: U \in \Gamma \rightarrow \Gamma$, where the neighborhood $U$ designates those points that return to $\Gamma$ at least once along the orbit of system (1). Thus, $P$ defines a 2-D discrete dynamical system $x_{k+1}=P\left(x_{k}\right), k=0,1, \ldots$, which characterizes system (1). For the case of homoclinic orbit (or heteroclinic orbit), this particular Poincaré map (called the Shil'nikov map) guarantees that the system is chaotic in a rigorous mathematical sense. In the following, we aim to give an analytical representation, based on experimental data of the homoclinic orbit in the Chen system with linear time-delay feedback.

\subsection{The part of the homoclinic orbit corresponding to the complex eigenvalues}

From Fig. 1, the homoclinic orbit consists of two parts: one part of the homoclinic orbit approaches to (departs from) the equilibrium point monotonically, which corresponds to the negative (positive) real eigenvalue; another part of the homoclinic orbit departs from (approaches to) the equilibrium point in an oscillatory way, corresponding to the complex eigenvalues with positive (negative) real part. The analytical 
solutions of the two parts are significantly different. Notice, however, that the previous methods do not distinguish between the two parts [Algaba et al., 2010, 2012, 2013a,b, 2014].

Here, we assume that the projection of the part of the orbit that corresponds to the complex eigenvalues with respect to a plane $I$ is a spiral involute. Plane $I$ is represented by $X_{1} O_{1} Y_{1}$ in the coordinate system $O_{1}$ with origin at the equilibrium point. If we know the coordinates of the plane $I$, we can convert any trajectory in $I$ into the original coordinate system by utilizing RPY(Roll-Pitch-Yaw) method about rotation angles. Thus, it is then possible to derive the part of the homoclinic orbit corresponding to the image eigenvalue in the original coordinates.

Suppose that the origin of coordinate of the new origin $O_{1}$ in the original coordinate system is $P_{0}\left(x_{0}, y_{0}, z_{0}\right)$. The rotation angles of the unit vector $\left(X_{1}, Y_{1}, Z_{1}\right)$ in the new coordinate system $O_{1}$ with respect to the $X, Y, Z$ axes of the original coordinate system $O$ are $\varepsilon x, \varepsilon y, \varepsilon z$, respectively. The rotation matrix from the $O_{1}$ coordinate system to the $O$ coordinate system is given by

$$
{ }_{O 1}^{O} R(\varepsilon x, \varepsilon y, \varepsilon z)=R(x, \varepsilon x) R(y, \varepsilon y) R(z, \varepsilon z),
$$

where

$$
\begin{aligned}
& R(x, \varepsilon x)=\left[\begin{array}{ccc}
1 & 0 & 0 \\
0 & \cos (\varepsilon x) & -\sin (\varepsilon x) \\
0 & \sin (\varepsilon x) & \cos (\varepsilon x)
\end{array}\right], \\
& R(y, \varepsilon y)=\left[\begin{array}{ccc}
\cos (\varepsilon y) & 0 & \sin (\varepsilon y) \\
0 & 1 & 0 \\
-\sin (\varepsilon y) & 0 & \cos (\varepsilon y)
\end{array}\right], \\
& R(z, \varepsilon z)=\left[\begin{array}{ccc}
\cos (\varepsilon z) & -\sin (\varepsilon z) & 0 \\
\sin (\varepsilon z) & \cos (\varepsilon z) & 0 \\
0 & 0 & 1
\end{array}\right] .
\end{aligned}
$$

By utilizing RPY method, an arbitrary point $f_{O 1}$ in the coordinate system $O_{1}$ transforming into a point $f_{O}$ in the coordinates system $O$ is

$$
f_{O}={ }_{O 1}^{O} R f_{O 1}+P_{0} .
$$

Using Eqs. (3)-(7), we obtain the normal vector $H=\left(A_{1}, A_{2}, A_{3}\right)$ of the plane $I$ in the coordinate system $O$. Then, the equation of plane $I$ that passes through the point $P_{0}\left(x_{0}, y_{0}, z_{0}\right)$ and with $H$ as a normal vector is given by

$$
A_{1} x+A_{2} y+A_{3} z+D=0
$$

where

$$
D=-A_{1} x_{0}-A_{2} y_{0}-A_{3} z_{0} .
$$

The standard spiral involute equation in plane $I$ is assumed to be

$$
\begin{aligned}
& x_{1}(t)=e^{\sigma t} \beta \cos (\omega t), \\
& y_{1}(t)=e^{\sigma t} \beta \cos \left(\omega t+\frac{\pi}{2}\right), \\
& z_{1}(t)=0,
\end{aligned}
$$

where $\beta$ denotes a constant of the distance between adjacent spirals in Eq. (10). The projection transformation from the original coordinate system $O$ to plane $I$ is given as

$$
\left\{\begin{array}{l}
x_{1}(t)=x(t)-A_{1} \Phi(t) \\
y_{1}(t)=y(t)-A_{2} \Phi(t) \\
z_{1}(t)=z(t)-A_{3} \Phi(t)
\end{array}\right.
$$


where $\Phi(t)=\frac{A_{1} x(t)+A_{2} y(t)+A_{3} z(t)+D}{A_{1}^{2}+A_{2}^{2}+A_{3}^{2}}$. Denote $\chi=A_{1}^{2}+A_{2}^{2}+A_{3}^{2}$. Then, we have

$$
\left[\begin{array}{l}
x_{1} \\
y_{1} \\
z_{1}
\end{array}\right]=N^{\dagger}\left[\begin{array}{l}
x(t) \\
y(t) \\
z(t)
\end{array}\right]-\left[\begin{array}{c}
\frac{D A_{1}}{\chi} \\
\frac{D A_{2}}{\chi} \\
\frac{D A_{3}}{\chi}
\end{array}\right]
$$

where $N=\left[\begin{array}{c}1-\frac{A_{1}^{2}}{\chi}-\frac{A_{1} A_{2}}{\chi}-\frac{A_{1} A_{3}}{\chi} \\ -\frac{A_{1} A_{2}}{\chi} 1-\frac{A_{2}^{2}}{\chi}-\frac{A_{2} A_{3}}{\chi} \\ -\frac{A_{1} A_{3}}{\chi}-\frac{A_{2} A_{3}}{\chi} 1-\frac{A_{3}^{2}}{\chi}\end{array}\right]^{\dagger}$, where the notation " $\dagger$ " is the inverse matrix for nonsingular matrix (or pseudo inverse matrix for singular matrix), $(x(t), y(t), z(t))$ is the part of the homoclinic orbit corresponding to the complex eigenvalues of the Chen system with linear time-delay feedback dependence of $x_{1}, y_{1}, z_{1}$ on time $t$. So, we have

$$
\left[\begin{array}{l}
x(t) \\
y(t) \\
z(t)
\end{array}\right]=N\left[\begin{array}{l}
x_{1}(t) \\
y_{1}(t) \\
z_{1}(t)
\end{array}\right]+N\left[\begin{array}{c}
\frac{D A_{1}}{\chi} \\
\frac{D A_{2}}{\chi} \\
\frac{D A_{3}}{\chi}
\end{array}\right]
$$

Finally, we get the equations of the part of homoclinic orbit corresponding to the complex eigenvalues in the coordinate system $O$, given as

$$
\left\{\begin{array}{l}
x(t)=N_{11}\left(x_{1}(t)+\frac{D A_{1}}{\chi}\right)+N_{12}\left(y_{1}(t)+\frac{D A_{2}}{\chi}\right)+N_{13}\left(z_{1}(t)+\frac{D A_{3}}{\chi}\right), \\
y(t)=N_{21}\left(x_{1}(t)+\frac{D A_{1}}{\chi}\right)+N_{22}\left(y_{1}(t)+\frac{D A_{2}}{\chi}\right)+N_{23}\left(z_{1}(t)+\frac{D A_{3}}{\chi}\right), \\
z(t)=N_{31}\left(x_{1}(t)+\frac{D A_{1}}{\chi}\right)+N_{32}\left(y_{1}(t)+\frac{D A_{2}}{\chi}\right)+N_{33}\left(z_{1}(t)+\frac{D A_{3}}{\chi}\right) .
\end{array}\right.
$$

\subsection{The part of the homoclinic orbit corresponding to the real eigenvalue}

For the part of the homoclinic orbit corresponding to the real eigenvalue, we use the scaling logarithm change series method proposed in [Bao \& Yang, 2011] and a modified undetermined coefficient method to get the analytical expression of this part of the homoclinic orbit.

For $t>0$, let

$$
t=\frac{1}{T} \ln (\eta)
$$

where $\eta$ is the new time variable, and $T$ is an undetermined positive real constant, referred to as scaling factor. Substituting (15) into (1) gives $\frac{d \mathbf{x}}{d \eta}=\mathbf{f}(\mathbf{x}) \frac{1}{T \eta}$, i.e.,

$$
\operatorname{T\eta } \frac{d \mathbf{x}}{d \eta}=\mathbf{f}(\mathbf{x})
$$

The equation of the part of the homoclinic orbit corresponding to the real eigenvalue is given by 


$$
\left\{\begin{array}{l}
x(t)=x_{0}+\sum_{k=1}^{\infty} a_{k} \eta^{k} \\
y(t)=y_{0}+\sum_{k=1}^{\infty} b_{k} \eta^{k} \\
z(t)=z_{0}+\sum_{k=1}^{\infty} c_{k} \eta^{k} \\
z(t-\tau)=z_{0}+\sum_{k=1}^{\infty} c_{k} e^{-T(t-\tau) k}
\end{array}\right.
$$

where $\left(x_{0}, y_{0}, z_{0}\right)=C_{+}$representing equilibrium, $a_{k}, b_{k}, c_{k}(k=1,2,3, \ldots, n)$ are undetermined coefficients. Substituting (17) into (16), we have

$$
\left\{\begin{array}{l}
T \eta \sum_{k=1}^{\infty} k a_{k} \eta^{k-1}=f_{i}\left(x_{0}+\sum_{k=1}^{\infty} a_{k} \eta^{k}, y_{0}+\sum_{k=1}^{\infty} b_{k} \eta^{k}, z_{0}+\sum_{k=1}^{\infty} c_{k} \eta^{k}\right) \\
T \eta \sum_{k=1}^{\infty} k b_{k} \eta^{k-1}=f_{i}\left(x_{0}+\sum_{k=1}^{\infty} a_{k} \eta^{k}, y_{0}+\sum_{k=1}^{\infty} b_{k} \eta^{k}, z_{0}+\sum_{k=1}^{\infty} c_{k} \eta^{k}\right) \\
T \eta \sum_{k=1}^{\infty} k c_{k} \eta^{k-1}=f_{i}\left(x_{0}+\sum_{k=1}^{\infty} a_{k} \eta^{k}, y_{0}+\sum_{k=1}^{\infty} b_{k} \eta^{k}, z_{0}+\sum_{k=1}^{\infty} c_{k} \eta^{k}\right) .
\end{array}\right.
$$

By comparing the coefficients of the term $\eta^{1}$ in (18), we have

$$
\left(T E+J\left(C_{+}\right)\right)\left(\begin{array}{c}
a_{1} \\
b_{1} \\
c_{1}
\end{array}\right)=0,
$$

where $J\left(C_{+}\right)$is the Jacobian of system evaluated at the equilibrium $C_{+}$, and $E$ is an identity matrix. By choosing parameter $T$ such that (19) has a non-trivial solution, we have $T$. Using the undetermined coefficients, we can determine all coefficients $a_{k}, b_{k}, c_{k}(k \geq 2)$ and $\beta$, which are completely determined by $a_{1}, b_{1}, c_{1}$.

After this intersection point $\left(x_{n}, y_{n}, z_{n}\right)$ is determined, according to the continuity of the orbits $\sum_{k=1}^{\infty} a_{k}=$ $x_{n}-x_{0}, \sum_{k=1}^{\infty} b_{k}=y_{n}-y_{0}, \sum_{k=1}^{\infty} c_{k}=z_{n}-z_{0}$, we can find $a_{1}, b_{1}, c_{1}$ and $\beta$. And then we can use the undetermined coefficient method to obtain the coefficients of equation (18), getting then the analytic solutions of homoclinic orbit.

Now we have to prove the convergence of the solution. Because $\sum_{k=1}^{\infty} a_{k}$ is bounded, there exists an $M>0$ such that $\sum_{k=1}^{\infty} a_{k} \leq M$. Consequently, $\sum_{k=1}^{\infty} a_{k} \eta^{k} \leq M \sum_{k=1}^{\infty} \eta^{k}=M \sum_{k=1}^{\infty} e^{T k t}$ is convergent on $t \in(0,+\infty)$. However, we have $\lim _{t \rightarrow+\infty} e^{T k t}=0$, so the series $x(t)$ is convergent to $x_{0}$. Similarly, the convergence of the series $y(t)$ and $z(t)$ can be shown.

Note that, the algorithms for computation of homoclinic orbit in the chaotic system in this work is sufficiently different from that in [Leonov, 2013b]. In [Leonov, 2013b], the authors proved that the existence of homoclinic orbit can be justified using the Fishing principle, which is based on the construction of a special two dimensional manifold so that the separatrix of the saddle of the system could intersect the manifold. From the Fishing principle, the homoclinic orbit can be obtained by a numerical procedure to approximate parameters, yet the analytical solution of the homoclinic orbit of the system has not been derived. Different from the method in [Leonov, 2013b], we first treat the different trajectories corresponding to real eigenvalue and image eigenvalues differently, which is the important contribution of this work; then, the analytical solution of the homoclinic orbit of the system is formulated in this paper; finally, we identify the homoclinic orbit and demonstrate it by the experimental data and numerical plot. 


\section{Homoclinic orbit of the single-scroll attractor in the Chen system with linear time-delay feedback}

To demonstrate the proposed method in this paper, we consider the Chen system with linear time-delay with a single-scroll attractor.

\subsection{The single-scroll attractor in the Chen system with linear time-delay feedback}

In 1999, Chen et.al report a new chaotic system, which resembles some familiar features from the Lorenz attractors, called Chen system [Chen \& Ueta, 1999]. The Chen system with linear time-delay feedback is given as [Ren \& Li, 2010; Ren et al., 2006]

$$
\left\{\begin{array}{l}
\dot{x}(t)=a(y(t)-x(t)) \\
\dot{y}(t)=(c-a) x(t)-x(t) z(t)+c y(t) \\
\dot{z}(t)=x(t) y(t)-b z(t)+k(z(t)-z(t-\tau))
\end{array}\right.
$$

where $a=35, b=3, c=18.35978, k$ is the time-delay feedback gain.

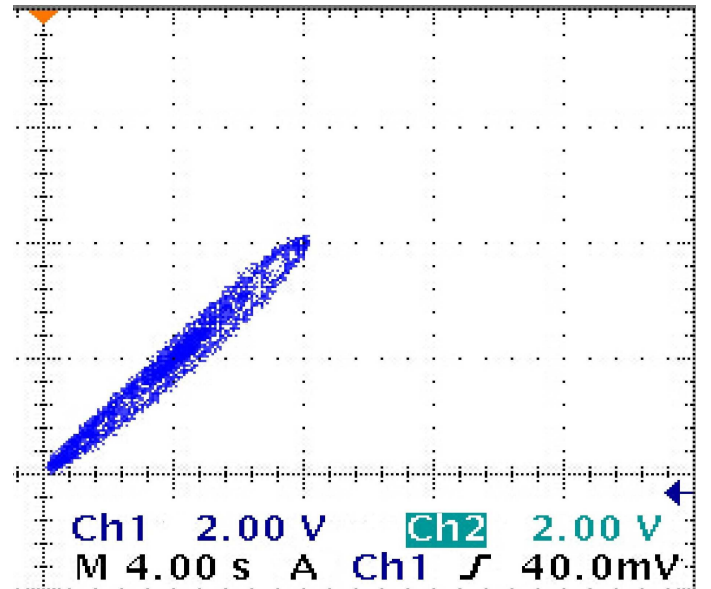

(a)

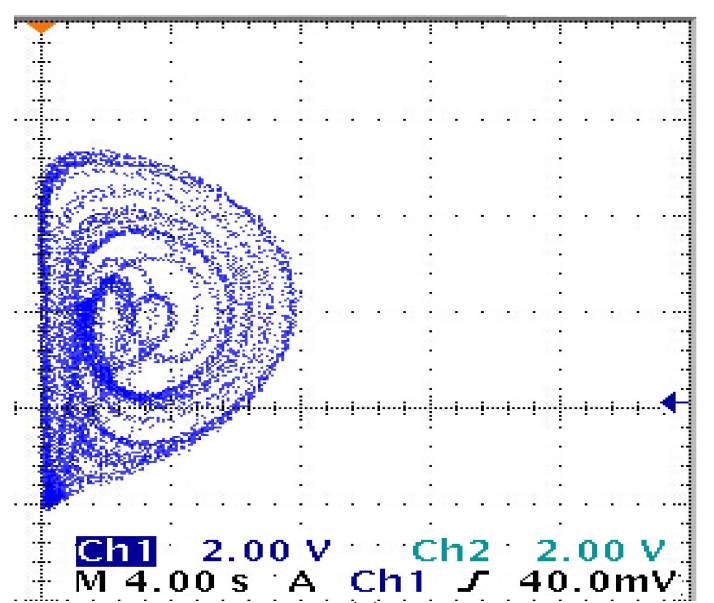

(c)

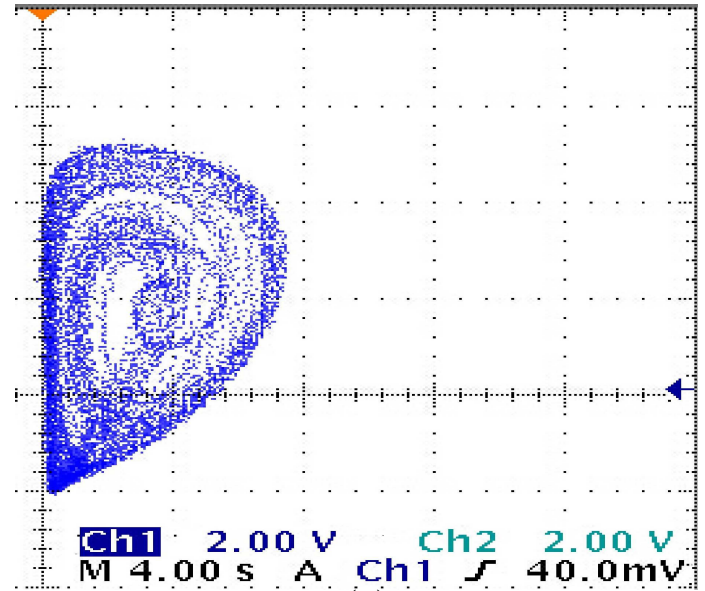

(b)

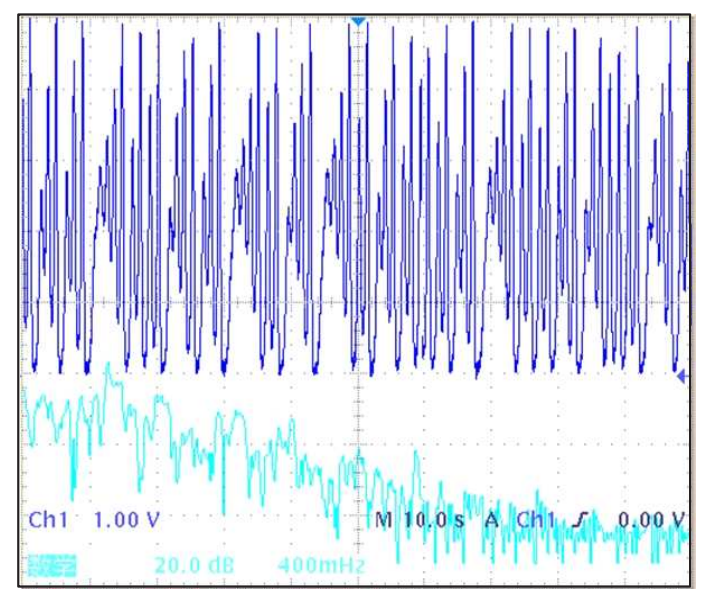

(d)

Fig. 2. The single-scroll attractor in the experimental Chen system with linear time-delay feedback, (a) trajectory in the $\mathrm{x}-\mathrm{y}$ plane, (b) trajectory in the $\mathrm{y}-\mathrm{z}$ plane, (c) trajectory in the $\mathrm{x}-\mathrm{z}$ plane, (d) time domain waveform of $\mathrm{x}$ and its power spectrum

The addition of linear time-delay feedback to the nonchaotic Chen system has been shown to produce 
double-scroll attractors [Ren \& Li, 2010; Ren et al., 2006] and multi-scroll attractor [Ren et al., 2017].

In this paper, we show that the Chen system with linear time-delay feedback also demonstrate a singlescroll attractor for parameters $k=2.85, \tau=0.3$. The experimental results of the single-scroll attractor are shown in Fig. 2. The initial condition of the attractor is $x(0)=2.27, y(0)=2.27, z(0)=1.72, z(t)=0$, when $-\tau \leq t \leq 0$. Figure $2(\mathrm{~d})$ shows that $x$-waveform obtained from the experiment is broadband. Our experimental circuit implementation of the Chen system with linear time-delay is given in the Appendix in detail.

The third equation of system (20) is a time delay differential equation, which can be converted into ordinary differential equation by using the method in [Ren et al., 2017]. Using the method to calculate finite-time local Lyapunov exponent spectrum in [Kuznetsov et al., 2018; Leonov \& Kuznetsov, 2007; Leonov et al., 2015b], we obtain the finite-time local Lyapunov exponent spectrum of the Chen system with linear time-delay. The two largest non-zero exponents are 0.8055 and 0.12 . In order to further show that the attractor generated by the Chen circuit with direct time-delay feedback is indeed chaotic, we apply the proposed method to identify the homoclinic orbit of the system and then use the Shil'nikov-type Lemma to show the existence of chaos.

\subsection{The existence of chaos in the Chen system with linear time-delay feedback}

The Shil'nikov theorem has been updated for high-dimensional systems according to the literature [Glendinning \& Tresser, 1985], which is referred to as Shil'nikov-type Lemma. For DDE systems (20), there exist an infinity of eigenvalues, as shown in Fig. 3. Complex eigenvalues are denoted as $\lambda_{i, j}=-\sigma_{i, j} \pm \mathbf{i} \omega_{i, j}$, $(i=1,2,3, \ldots ., n ; j=1,2,3, \ldots ., n)$ (where $\sigma_{i, j}>0$ and $\left.\omega_{i, j} \neq 0\right)$ and real number eigenvalues are denoted as $\gamma_{d i}(d i=n+1, n+2, \ldots)$, the Shil'nikov-type Lemma is given as follows.

Shil'nikov-type Lemma(Homoclinic orbits)[Glendinning \& Tresser, 1985]: Suppose that $O_{+}$is a saddle focus whose eigenvalues satisfy conditions (i)-(iii):

(i) $\lambda_{i, j}=-\sigma_{i, j} \pm \mathbf{i} \omega_{i, j}, \quad\left(\sigma_{i, j}>0\right), \quad \gamma_{d i}>0 \quad(d i=n+1), \quad \gamma_{d i} \leq 0 \quad(d i=n+2, \ldots), \quad$ and $\quad \gamma_{d i}>\sigma_{i, j}$, $2 \sigma_{i, j}+\gamma_{d i}<0$

(ii) $\lambda_{i, j}=\sigma_{i, j} \pm \mathbf{i} \omega_{i, j},\left(\sigma_{i, j}>0\right), \gamma_{d i} \leq 0(d i=n+1, n+2, \ldots)$, and $\gamma_{d i}<-\sigma_{i, j}, 2 \sigma_{i, j}+\gamma_{i d}<0$;

(iii) $\lambda_{i, j}= \pm \sigma_{i, j} \pm \mathbf{i} \omega_{i, j}\left(\sigma_{i, j}>0\right)$, no $\gamma_{d i}$ exists, and all $\sigma_{i, j}$ are not equal to each other,

and there exists a homoclinic orbit that connects $O_{+}$to itself, then the Shil'nikov map, defined in a neighborhood of the homoclinic orbit that connects $\mathrm{O}_{+}$, has a countable number of Smale horseshoes in its dynamics and the system exhibits chaos.

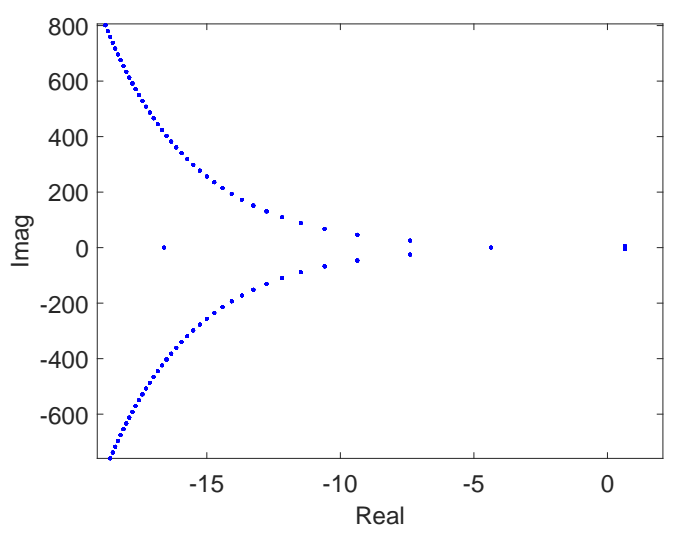

Fig. 3. Characteristic roots for the equilibrium $O_{+}$

The Lemma provides a way to show the existence of chaos in an experimental attractor as that demonstrated (by Fig. 2) in the Chen system with linear time-delay.

The Lemma can be applied for the single-scroll attractor of Chen system with linear time-delay feedback because of the following: 
1) $C_{+}$is a hyperbolic saddle focus:

System (20) has three equilibria:

$O_{0}:(0,0,0) ; O_{+}:\left(x_{0}, y_{0}, z_{0}\right) ; O_{-}:\left(-x_{0},-y_{0}, z_{0}\right)$, where $x_{0}=y_{0}=\sqrt{b(2 c-a)}, z_{0}=2 c-a$. The characteristic equation at $O_{+}$is

$$
\lambda^{3}+(a+b-c-k) \lambda^{2}+[b c+c k-a k] \lambda+4 a b c-2 a^{2} b+\left[\lambda^{2}+(a-c) \lambda\right] k e^{-\lambda \tau}=0 .
$$

For the typical parameters set: $a=35, b=3, c=18.35978, k=2.85, \tau=0.3$, the real eigenvalues are given by $\gamma_{1}=-4.3578, \gamma_{2}=-16.60$, and the two of the complex eigenvalues are given by $\sigma_{1,2} \pm \mathbf{i} \omega_{1,2}=0.6517 \pm \mathbf{i} 5.7533$, therefore, $O_{+}$is a hyperbolic saddle focus. The second condition of the Shil'nikov-type Lemma criterion is satisfied.

2) There exists a homoclinic orbit passing $O_{+}$:

For system (20) with typical parameters, according to the method in Sec. 2, let $P_{0}=(2.271277$, $2.271277,1.71956), \varepsilon x=\pi / 4.35, \varepsilon y=\pi / 4.35, \varepsilon z=\pi / 1.998$. We obtain the part of homoclinic orbit corresponding to the complex eigenvalues.

In general, the domains $V^{+}$and $V^{-}$exist in the stable invariant manifold $W_{l o c}^{s}$ and in the unstable invariant manifold $W_{l o c}^{u}$, respectively.If the stable and unstable manifolds intersect, then this intersection must be non-transverse; their intersection must lie on both manifolds. Definition of the intersection set $M^{+}\left\{(x, y, z):(x, y, z) \in V^{+} \cap V^{-}\right\}$within the invariant set of both stable and unstable manifolds of the dynamical system [Shil'nikov et al., 1998], if it exists, will drift into the equilibrium. In this paper, $M^{+}$ was obtained through experiments, as shown in Fig. 4. We define $\xi\left(x_{n}, y_{n}, z_{n}\right) \in M^{+}$as the intersection point.

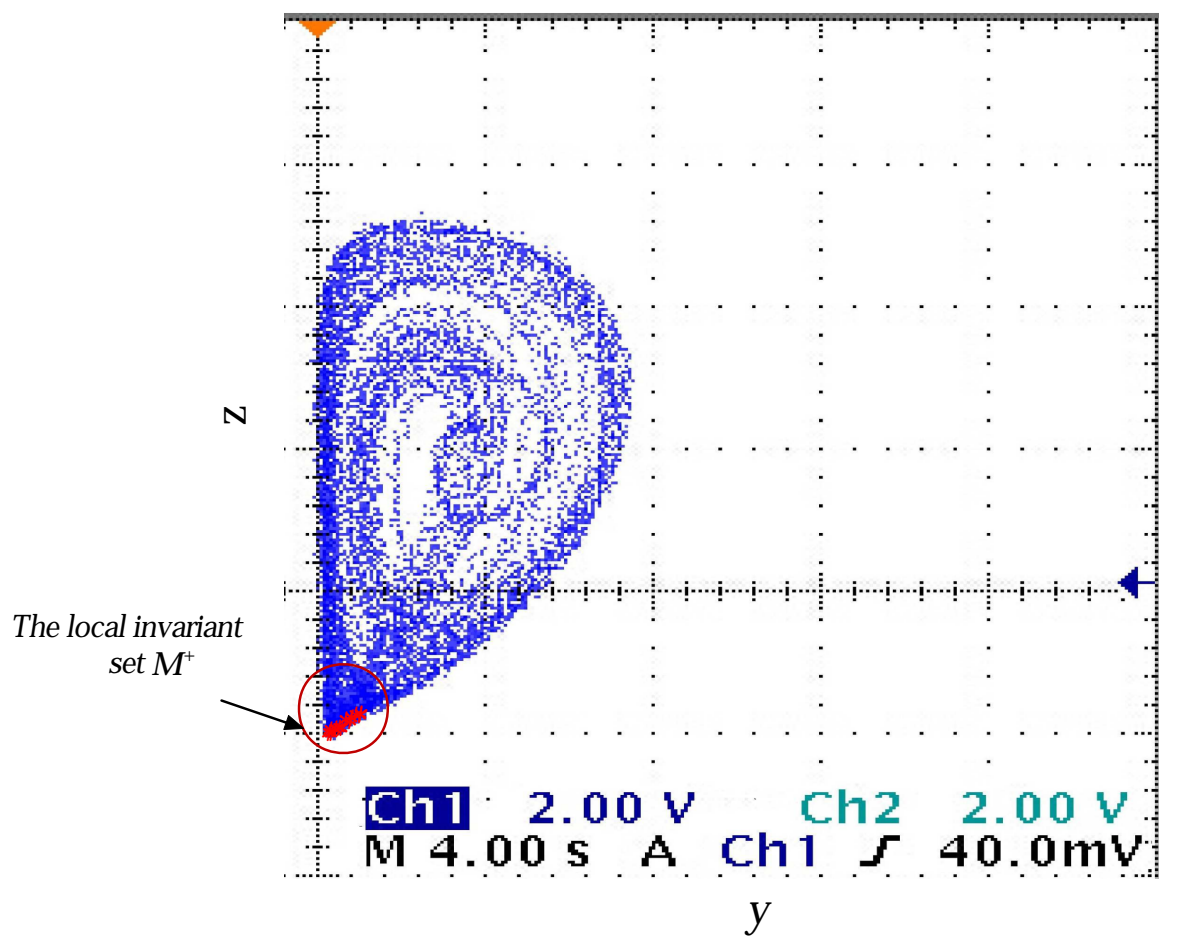

Fig. 4. The single-scroll attractor in the experimental Chen system with linear time-delay feedback trajectory in the $y$ - $z$ plane, the experimental result from the observed data 

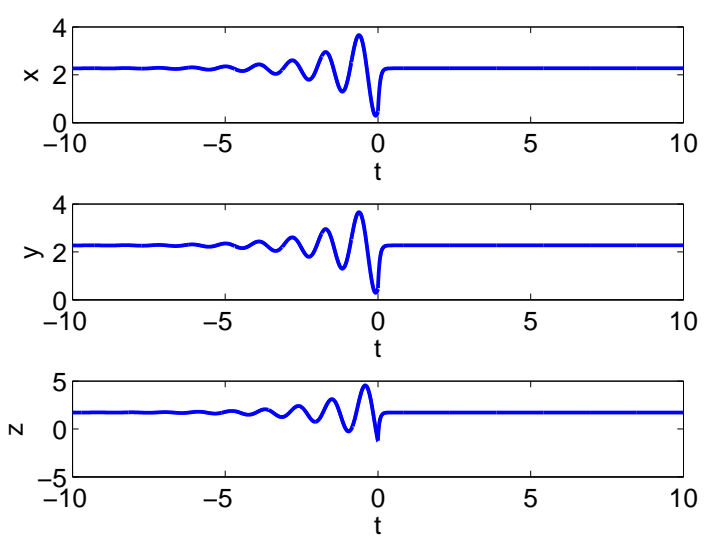

Fig. 5. Trajectories of $x, y, z$ of the homoclinic orbit of Fig. 6. Visualization of 3-D homoclinic orbit of the Chen the Chen system with linear time-delay feedback

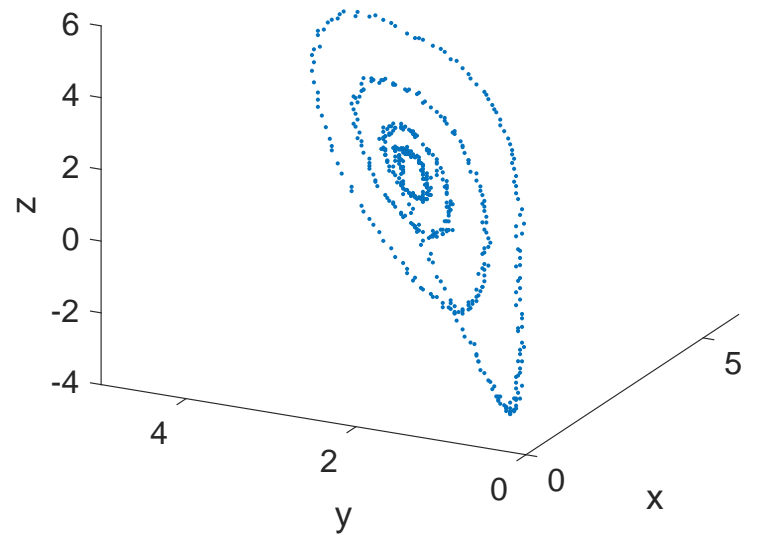

system with linear time-delay feedback using experiment data

The intersection point is given as $\xi=(0.3491,0.4002,-1.5145)$, then we have $T=-17.5288, \beta=2.295$, $A_{1}=0.661, A_{2}=-0.496, A_{3}=0.563, D=-1.343, a_{1}=-1.968, b_{1}=-1.7814, c_{1}=-2.3857$. According to these parameters, the analytic solutions of homoclinic orbit are obtained. The time domain waveform and phase plot of the homoclinic orbit are given in Figs. 4 and 5, respectively.

To this end, the homoclinic orbit approaching $O_{+}$in both time forward and backward direction are obtained from the experiment. The eigenvalues of $O_{+}$satisfy condition (ii) in the Shilnikov-type Lemma. In conclusion, we experimentally validate the presence of a homoclinic orbit in the Chen system with linear time-delay feedback. Furthermore, according to the Shil'nikov criterion, the Chen system with linear time-delay feedback does have a Smale horseshoes and, hence, chaos.

Since $O_{-}$and $O_{+}$have similar properties, there also exists a homoclinic orbit across $O_{-}$. Therefore, it also has chaos at $O_{-}$, which is symmetrical to the attractor given in Fig. 2.

\subsection{Method extension to the generalized Lorenz-like system with linear time-delay}

The generalized Lorenz-like system with time-delay is given by

$$
\left\{\begin{array}{l}
\dot{x}=\sigma(y-x), \\
\dot{y}=r x-d y-x z, \\
\dot{z}=x y-b z+k[z-z(t-\tau)],
\end{array}\right.
$$

where $\sigma, r, d, b$, and $k$ are parameters.

The aforementioned Chen system with linear time-delay as given by Eq. (20) is one case of this generalized Lorenz-like system. To see this point, using the following transformation [Leonov \& Kuznetsov , 2015]

$$
x \rightarrow-h X, y \rightarrow-h Y, z \rightarrow-h Z, \psi=-h t,
$$


when $h=c$, the Chen system with linear time-delay in Eq. (20) becomes

$$
\left\{\begin{array}{l}
\frac{d X}{d \psi}=-\frac{a}{c}(Y-X), \\
\frac{d Y}{d \psi}=\left(\frac{a}{c}-1\right) X-Y-X Z, \\
\frac{d Z}{d \psi}=\frac{b}{c} Z+X Y-\frac{k}{c}[Z-Z(t-\tau)] .
\end{array}\right.
$$

Considering the Lorenz system with linear time-delay

$$
\left\{\begin{array}{l}
\frac{d X}{d t}=\sigma(Y-X) \\
\frac{d Y}{d t}=\rho X-X Z-Y \\
\frac{d Z}{d t}=X Y-\beta Z+K(Z(t)-Z(t-\tau))
\end{array}\right.
$$

we know that the two systems are homothetic copies [Algaba et al., 2013c] by replacing parameters in Eq.(25) with

$$
\sigma=-\frac{a}{c}, \rho=\frac{a}{c}-1, \beta=-\frac{b}{c}, K=-\frac{k}{c} .
$$

Therefore, for $c \neq 0$, the Chen system with linear time delay in (24) is equivalent to the Lorenz system with linear time-delay in the particular case where the parameter is $\rho+\sigma=-1$.

The method to identify the homoclinic orbit in this Section can be used in the generalized Lorenz-like system with linear time-delay, including the Lu system, the Chen system and the Lorenz system all with time delay. All these systems can be transformed to the same algebraic form by a transformation with the inversion of time, and such transformation preserves the homoclinic orbit [Leonov \& Kuznetsov , 2015].

\section{Conclusions}

In this paper, we first propose an analysis for the existence of a homoclinic orbit by using the spiral hypothesis and improved undetermined coefficients methods, whose method is a general method to find the homoclinic orbit; it can be easily extended to the Lorenz-like systems. Then, we generate experimentally a single-scroll hyper-chaotic attractor from the Chen circuit with linear time-delay feedback, identifying the homoclinic orbit from data. Finally, we use the Shil'nikov-type Lemma to show that the experimental single-scroll attractor is indeed chaotic.

\section{Acknowledgments}

The work is supported in part by National Natural Science Foundation of China (60804040, 61172070), Scientific and Technological Innovation Leading Talents Program of Shaanxi Province, Key Program of Natural Science Fund of Shaanxi Province(2016ZDJC-01).

\section{Appendices}

\section{Appendix A}


In order to get the single-scroll attractor experimentally, we build a circuit to implement the time-delay. The existing methods for circuit implementation of time-delay include the delay-line method, which can generate very high and ultra-high frequency hyperchaotic oscillations [Mykolaitis, 2003], a T-type LCL network [Namajunas et al., 1995], a digital sampling and replying with memory shift method [Wang et al., 2001; Ren \& Li, 2010], the all-pass filter method [Hu, 2009], and chains of $n$ Bessel filters [Buscarino et al., 2011]. In this paper, we use cascaded time-lag units to realize time-delay. Compared with the other methods, our proposed method is simpler in circuit implementation. The circuit of time-lag unit used is shown in Fig. A.1.

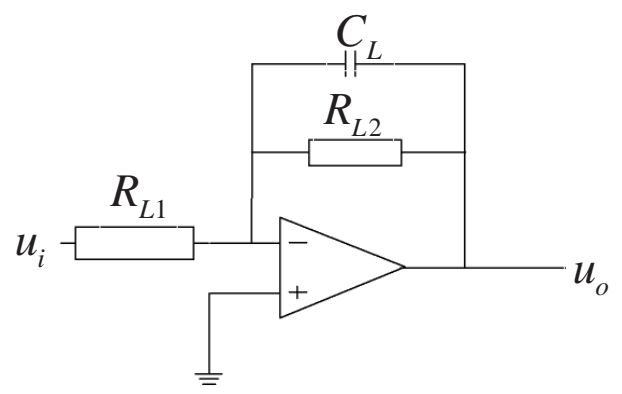

Fig. A.1. The time-lag unit.

The transfer function of the time-lag unit is:

$$
G(s)=\frac{K}{\bar{T} s+1}=\frac{R_{L 2}}{R_{L 1}\left(1+R_{L 2} C_{L} s\right)},
$$

where $\bar{T}=R_{L 2} C_{L}$ is the time constant and $K=\frac{R_{L 2}}{R_{L 1}}$ is gain ${ }^{1}$. Amplitude-frequency and phase-frequency characteristics of the unit are

$$
|G(j \omega)|=\frac{K}{\sqrt{1+(\bar{T} \omega)^{2}}}, \angle G(j \omega)=-\arctan (\bar{T} \omega) .
$$

By using $n$ time-lag units cascade, we obtain

$$
\left|G^{\prime}(j \omega)\right|=\left(\frac{K}{\sqrt{1+(\bar{T} \omega)^{2}}}\right)^{n}, \angle G^{\prime}(j \omega)=-n \arctan (\bar{T} \omega),
$$

where $\bar{T}=\frac{\tau}{n}$. If $n$ is large enough, then $\bar{T}$ is small enough, therefore we have $\left|G^{\prime}(j \omega)\right| \approx K^{n}$, $\angle G^{\prime}(j \omega) \approx-\tau \omega$.

When the time constant is small enough, the time-lag units can be approximated as a pure delay. The cascaded time-lag units can thus realize the time-delay $\tau$ approximately. As $n$ is increased, a more accurate approximation is achieved, but a larger number of units is needed. In our experiment, we use 15 time-lag units to approximate the time-delay 0.3 after. Because the amplitude-frequency characteristics of the time-lag unit is not 1 , we can add a proportional element in the cascade circuit to compensate the amplitude frequency characteristics, so as to make the amplitude of the cascaded units to be 1.

The schematic diagram of 15 cascaded time-lag units realizations of the time-delay is shown in Fig. A.2, where an operational amplifier at the right corresponds to the amplitude compensation unit which guarantees the gain of the signal to be equal to 1 . In Fig. A.2, there are 13 cascaded identical time-lag units between the first one and the fifteenth one, that are represented by the notation "...".

The schematic diagram of the Chen system is shown in Fig. A.3, where $R_{1}=R_{2}=R_{3}=R_{4}=R_{6}$ $=R_{7}=R_{13}=R_{14}=R_{16}=R_{17}=R_{19}=R_{21}=R_{22}=R_{23}=R_{24}=10 \mathrm{k} \Omega, R_{5}=2.86 \mathrm{M} \Omega, R_{8}=16500 \Omega, R_{9}=3 k \Omega, R_{10}=$

${ }^{1}$ The phase inverter is ignored at the output of the operational amplifier 


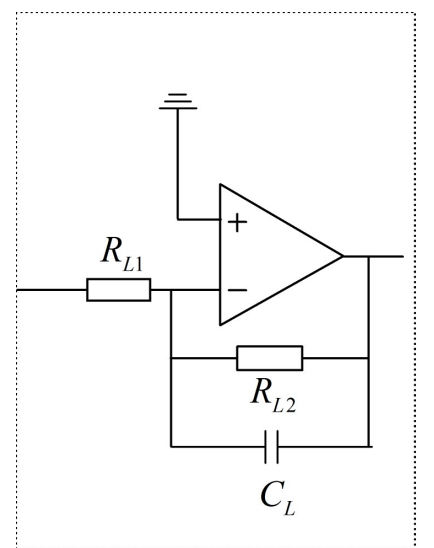

the first time lag unit

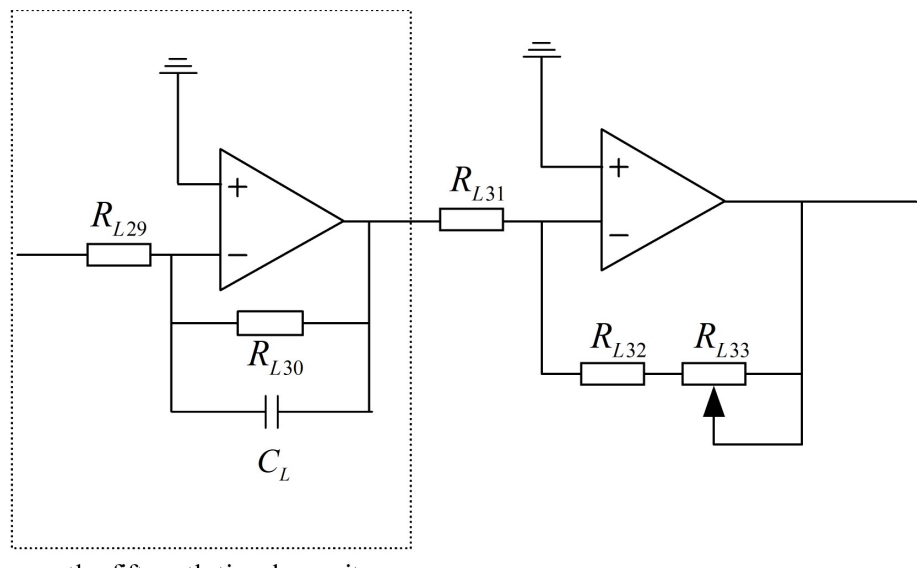

the fifteenth time lag unit

Fig. A.2. the schematic diagram of the time-delay implementation circuit.

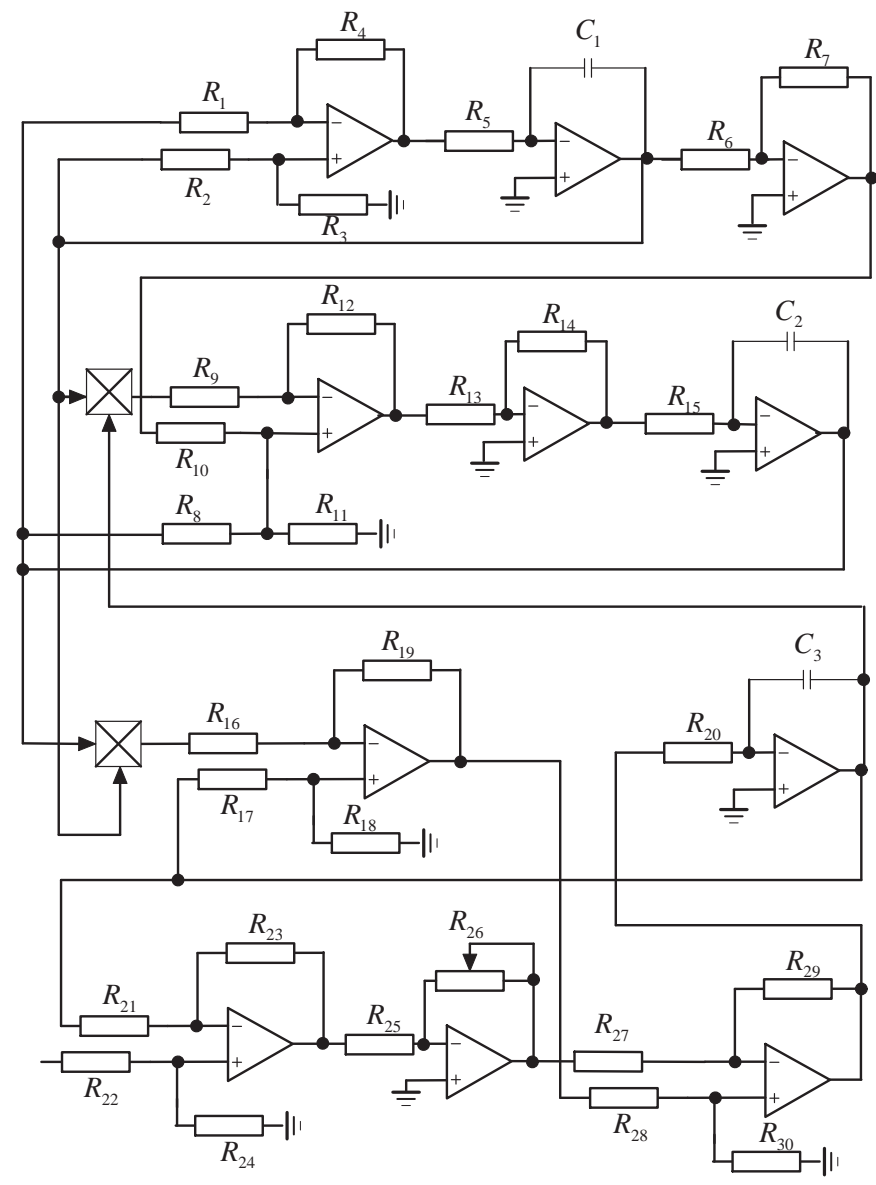

Fig. A.3. The schematic diagram of the Chen system.

$18215 \Omega, R_{11}=60000 \Omega, R_{12}=1000 \Omega, R_{15}=3.33 M \Omega, R_{18}=1765 \Omega, R_{20}=10 M \Omega, R_{25}=1 k \Omega, R_{27}=R_{28}=R_{29}=$ $R_{30}=10 k \Omega, C_{1}=C_{2}=C_{3}=10000 \mathrm{pF}, R_{26}=285 \Omega, R_{26}$ determines the delay feedback gain $k$ of system (20).

According to the circuit schematic diagram, the circuit equation is given in Equation (A.4) (the circuit design refers to [Zhong \& Tang, 2002; Ren \& Li, 2010]), 


$$
\left\{\begin{aligned}
\dot{x}= & \frac{-R_{3}\left(R_{1}+R_{4}\right)}{R_{1} R_{5} C_{1}\left(R_{2}+R_{3}\right)} x+\frac{R_{4}}{R_{1} R_{5} C_{1}} y \\
\dot{y}= & R_{14} R_{11}\left(R_{9}+R_{12}\right) \frac{-R_{7} R_{8} x+R_{6} R_{10} y}{R_{6} R_{9} R_{13} R_{15} C_{2}\left(R_{8} R_{11}+R_{10} R_{11}+R_{8} R_{10}\right)}-\frac{R_{14} R_{12}}{R_{9} R_{13} R_{15} C_{2}} x z \\
\dot{z}= & -\frac{R_{30}\left(R_{27}+R_{29}\right)}{R_{20} R_{27} C_{3}\left(R_{30}+R_{28}\right)}\left(\frac{R_{18}\left(R_{16}+R_{19}\right)}{R_{16}\left(R_{17}+R_{18}\right)} z-\frac{R_{19}}{R_{16}} x y\right) \\
& +\frac{R_{29}}{R_{27} R_{20} C_{3}}\left(-\frac{R_{24} R_{26}\left(R_{21}+R_{23}\right)}{R_{21} R_{25}\left(R_{22}+R_{24}\right)} z_{d}+\frac{R_{23} R_{26}}{R_{21} R_{25}} z\right)
\end{aligned}\right.
$$

where $z_{d}$ represents $z(t-\tau)$. The circuit parameters are

$$
\begin{aligned}
& \frac{R_{3}\left(R_{1}+R_{4}\right)}{R_{1} R_{5} C_{1}\left(R_{2}+R_{3}\right)}=\frac{R_{4}}{R_{1} R_{5} C_{1}}=a \\
& R_{14} R_{11}\left(R_{9}+R_{12}\right) \frac{-R_{7} R_{8}}{R_{6} R_{9} R_{13} R_{15} C_{2}\left(R_{8} R_{11}+R_{10} R_{11}+R_{8} R_{10}\right)}=c-a \\
& R_{14} R_{11}\left(R_{9}+R_{12}\right) \frac{R_{6} R_{10}}{R_{6} R_{9} R_{13} R_{15} C_{2}\left(R_{8} R_{11}+R_{10} R_{11}+R_{8} R_{10}\right)}=c \\
& \quad \frac{R_{30}\left(R_{27}+R_{29}\right)}{R_{20} R_{27} C_{3}\left(R_{30}+R_{28}\right)} \frac{R_{18}\left(R_{16}+R_{19}\right)}{R_{16}\left(R_{17}+R_{18}\right)}=b \\
& \frac{R_{29}}{R_{20} R_{27} C_{3}} \frac{R_{23} R_{26}}{R_{21} R_{25}}=\frac{R_{29}}{R_{27} R_{20} C_{3}} \frac{R_{24} R_{26}\left(R_{21}+R_{23}\right)}{R_{21} R_{25}\left(R_{22}+R_{24}\right)}=k
\end{aligned}
$$

By adding linear time-delay feedback to the non-chaotic Chen circuit, with feedback parameters $k=2.85, \tau=0.3$, we get experimentally a single-scroll attractor as shown in Fig. 2 in the main text. The results indicate that the linear time-delay feedback can produce a single-scroll attractor from the nonchaotic Chen system. The above time-delay experimental implementation method using cascade time-lag units is simpler and easier to integrate than the other methods.

\section{References}

Algaba, A., Fernandez-Sanchez, F., Merino, M. \& Rodriguez-Luis, A. J. [2010] "Comment on Heteroclinic orbits in Chen circuit with time delay," Communications in Nonlinear Science and Numerical Simulation, 17, 2708-2710.

Algaba, A., Fernandez-Sanchez, F., Merino, M. \& Rodriguez-Luis, A. J. [2012] "Comment on Existence of heteroclinic orbits of the Shil'nikov type in a 3D quadratic autonomous chaotic system," Journal of Mathematical Analysis and Applications, 329, 99-101.

Algaba, A., Fernandez-Sanchez, F., Merino, M. \& Rodriguez-Luis, A. J. [2013a] "Comment on Shil'nikovtype orbits of Lorenz-family systems," Physica A, 329, 4252-4257.

Algaba, A., Fernandez-Sanchez, F., Merino, M. \& Rodriguez-Luis, A. J. [2013b] "On Shil'nikov analysis of homoclinic and heteroclinic orbit of the T system," Journal of Computational and Nonlinear Dynamics, 8, 027001-4.

Algaba, A., Fernandez-Sanchez, F., Merino, M. \& Rodriguez-Luis, A. J. [2013c] "Chens attractor exists if Lorenz repulsor exists: The Chen system is a special case of the Lorenz system," Chaos, 23, 033108.

Algaba, A., Fernandez-Sanchez, F., Merino, M. \& Rodriguez-Luis, A. J. [2014] "Comment on Existence of heteroclinic orbits in two different chaotic dynamical systems," Applied Mathematics and Computation, 244, 49-56.

Algaba, A., Freire, E., Gamero, E. \&, Luis, R. A. J. [2015] "An exact homoclinic orbit and its connection with the Rössler system," Physics Letters A, 379, 1114-1121. 
Balasuriya, S. \& Padberg-Gehle, K. [2014] "Nonautonomous control of stable and unstable manifolds in two-dimensional flows," Physica D, 276, 48-60.

Bao, J. H. \& Yang, Q. G. [2011] "A new method to find homoclinic and heteroclinic orbits," Applied Mathematic and Computation, 217, 6526-6540.

Buscarino, A., Fortuna, L., Frasca, M. \&, Sciuto, G.. [2011] "Design of Time-Delay Chaotic Electronic Circuits," IEEE Transactions on Circuits and SystemsI: Regular papers, 58, 1888-1896.

Chen, G. R. \& Ueta, T. [1999] "Yet another chaotic attractor," International Journal of Bifurcation and Chaos, 9, 1465-1466.

Chen, G. W. [2013] "Non-periodic damped vibration systems with sublinear terms at infinity: Infinitely many homoclinic orbits," Nonlinear Analysis, 92, 168-176.

Chen, Y. Y., Chen, S. H. \& Zhao, W. [2017] "Constructing explicit homoclinic solution of oscillators: An improvement for perturbation procedure based on nonlinear time transformation," Communications in Nonlinear Science and Numerical Simulation, 48, 123-139.

Costa, D. G. \& Tehrani, H. [2014] "On a class of singular second-order Hamiltonian systems with infinitely many homoclinic solutions," Journal of Mathematical Analysis \&3 Applications, 412, 200-211.

Dong, C. W. \& Lan, Y. H. [2014] "A variational approach to connecting orbits in nonlinear dynamical systems," Physics Letters A, 378, 705-712.

EI-Dessoky, M. M., Yassen, M. T., Saleh, E. \& Aly, E. S. [2012] "Existence of heteroclinic orbits in two different chaotic dynamical systems," Applied Mathematics and Computation, 218, 11859-111870.

Glendinning, P. \& Tresser, C.[1985] "Heteroclinic loops leading to hyperchaos," Journal de Physique Letters, 46, 347-352.

Hu, G. S. [2009] "Hyperchaos of high order and its circuit implementation," International Journal of Circuit Theory and Applications, 39, 79-89.

Huang, Y. \& Yang, X. S. [2005] "Horseshoes in modified Chen's attractors," Chaos, Solitons E Fractals, 26, $79-85$.

Huaraca, W. \& Mendoza, V. [2016] "Minimal topological chaos coexisting with a finite set of homoclinic and periodic orbits," Physica D, 315, 83-89.

Kuznetsov, N. V., Leonov, G. A., Mokaev, T. N., Prasad A. \& Shrimali M. D. [2018] "Finite-time Lyapunov dimension and hidden attractor of the Rabinovich system," Nonlinear Dynamics, 92, 267-285.

Leonov, G. A, Kuznetsov, N. V.. [2007] "Time-Varying linearization and the Perron effects," International Journal of Bifurcation and Chaos, 17, 1079-1107.

Leonov, G. A. [2013a] "The Tricomi problem on the existence of homoclinic orbits in dissipative systems," Journal of Applied Mathematics and Mechanics, 77, 296-304.

Leonov, G. A. [2013b] "Shilnikov chaos in Lorenz-like systems," International Journal of Bifurcation and Chaos, 23, 1350058.

Leonov, G. A. \& Kuznetsov, N. V. [2015] "On differences and similarities in the analysis of Lorenz, Chen, and Lu system," Applied Mathematics and Computation, 256, 334-343.

Leonov, G. A., Kuznetsov, N. V. \& Mokaev, T. N. [2015a] "Hidden attractor and homoclinic orbit in Lorenz-like system describing convective fluid motion in rotating cavity," Communications in Nonlinear Science and Numerical Simulation, 28, 166-174.

Leonov G. A., Kuznetsov N. V. \& Mokaev T. N. [2015b] "Homoclinic orbits, and self-excited and hidden attractors in a Lorenz-like system describing convective fluid motion," Eur. Phys. J. Special Topics, 224, 1421-1458.

Li, Z. B., Tang, J. S. \& Cai, P. [2013] "A generalized harmonic function perturbation method for determining limit cycles and homoclinic orbits of Helmholtz-Duffing oscillator," Journal of Sound and Vibration, 332, 5508-5522.

Lima, M. F. S. \& Teixeira, M. A. [2013] "Homoclinic orbits in degenerate reversible-equivariant systems in $R^{6}$.," Journal of Mathematical Analysis and Applications , 403, 155-166.

Lin, X. B., Long, B. \& Zhu, C. R. [2015] "Multiple transverse homoclinic solutions near a degenerate homoclinic orbit," J. Differential Equations, 259, 1-24.

Lv, Y. \& Tang, C. L. [2013] "Homoclinic orbits for second-order Hamiltonian systems with subquadratic potentials," Chaos, Solitons \& Fractals, 57, 137-145. 
Mykolaitis, G. [2003] "Very high and ultrahigh frequency hyperchaotic oscillators with delay line," Chaos, Solitons \& Fractals, 17, 343-347.

Namajunas, A., Pyragas, K. \& Tamasevivius, A. [1995] "An electronic analog of the Mackey-Glass system," Physics Letters A, 201, 42-46.

Ren, H. P., Liu, D. \& Han, C. Z. [2006] "Anticontrol of chaos via direct time delay feedback," Acta Physica Sinica (in Chinese), 55, 2694-2701.

Ren, H. P. \& Li, W. C. [2010] "Heteroclinic orbits in Chen circuit with time delay," Communications in Nonlinear Science and Numerical Simulation, 15, 3058-3066.

Ren, H. P., Bai, C., Tian, K. \& Grebogi, C. [2017] "Dynamics of delay induced composite multi-scroll attractor and its application in encryption," International Journal of Non-Linear Mechanics, 94, $334-342$.

Shil'nikov, L. P. [1965] "A case of the existence of a countable number of periodic motions," Sov. Math. Docklady, 6, 163-166.

Shil'nikov, L. P., Shil'nikov, A. L., Turaev, D. V. \& Chua, L. O. [1998] "Method of Qualitative Theory in nonlinear dynamics (Part I)," World Scientific Series on Nonlinear Science Series A, 4.

Silva, C. P. [1993] "Shil'nikov theorem-a tutorial," IEEE Transactions on Circuits Systems-I, 40, 675-682.

Tucker, W. [1999] "The Lorenz attractor exists," Sciences, 328, 1197-1202.

Wang, J. W., Zhao, M. C. \& Zhang, Y. B. [2007] "Shil'nikov-type orbits of Lorenz-family systems," Physica $A, \mathbf{3 7 5}, 438-466$.

Wang, X. F., Zhong, G. Q. \& Tang, K. S. [2001] "Generating Chaos in Chua's circuit via time-delay feedback," IEEE. Trans. Circuits and Systems-I, 48, 1151-1156.

Xie, L. L. [2014] "A discussion on the coexistence of heteroclinic orbit and saddle foci for third-order systems," Journal of Mathematical Analysis and Applications, 412, 878-894.

Zheng, Z. H. \& Chen, G. R. [2006] "Existence of heteroclinic orbits of the Shil'nikov type in a 3D quadratic autonomous chaotic system," Journal of Mathematical Analysis Applications, 315, 106-119.

Zhong, G. Q. \& Tang, W. K. S.[2002] "Circuitry implementation and synchronization of Chen's attractor," International Journal of Bifurcation and Chaos, 12, 1423-1427.

Zhou, T. S., Tang, Y. \& Chen, G. R. [2004] "Chen's attractor exists," International Journal of Bifurcation and Chaos, 14, 3167-3177. 